\title{
Proton beam reirradiation for locally recurrent pancreatic adenocarcinoma
}

\author{
Pamela J. Boimel ${ }^{1}$, Abigail T. Berman ${ }^{1}$, Jonathan Li $^{1}$, Smith Apisarnthanarax ${ }^{2}$, Stefan Both ${ }^{3}$, Kristi \\ Lelionis $^{1}$, Gary L. Larson ${ }^{4}$, Ursina Teitelbaum ${ }^{5}$, John N. Lukens ${ }^{1}$, Edgar Ben-Josef ${ }^{1}$, James M. Metz ${ }^{1}$, \\ John P. Plastaras ${ }^{1}$
}

${ }^{1}$ Department of Radiation Oncology, University of Pennsylvania, Philadelphia, USA; ${ }^{2}$ Department of Radiation Oncology, University of Washington, Seattle, USA; ${ }^{3}$ Department of Radiation Oncology Memorial Sloan Kettering Cancer Center, New York, USA; ${ }^{4}$ Procure Proton Therapy Center, Oklahoma City, USA; ${ }^{5}$ Department of Hematology Oncology, University of Pennsylvania, Philadelphia, USA

Contributions: (I) Conception and design: PJ Boimel, JP Plastaras; (II) Administrative support: JP Plastaras, K Lelionis; (III) Provision of study materials or patients: S Apisarnthanarax, E Ben-Josef, U Teitelbaum, JM Metz, S Both, GL Larson, JP Plastaras; (IV) Collection and assembly of data: PJ Boimel, J Li, K Lelionis; (V) Data analysis and interpretation: PJ Boimel; (VI) Manuscript writing: All authors; (VII) Final approval of manuscript: All authors.

Correspondence to: Pamela J. Boimel, MD, PhD. University of Pennsylvania School of Medicine, TRC 2-West, 3400 Civic Center Boulevard, Philadelphia, PA 19104, USA. Email: Pamela.Boimel@uphs.upenn.edu.

Background: Local recurrence following definitive treatment for pancreatic adenocarcinoma is common and can be associated with significant morbidity and mortality. Retreatment options for these patients are limited. Proton beam reirradiation (PRT) may limit dose and toxicity to previously irradiated normal tissues in patients without evidence of metastatic disease.

Methods: Between 8/2010-2/2015, 15 patients with isolated, locally-recurrent pancreatic cancer were treated with PRT. Acute toxicity was graded using CTC v 4.0 and defined as occurring within 90 days. Kaplan-Meier survival analysis was performed from the start of PRT. A log-rank test was used to compare survival with or without concurrent chemotherapy.

Results: Median follow-up was 15.7 months [2-48] from the start of PRT. The median clinical target volume (CTV) was 71 cc [15-200]. Ten (67\%) patients received concurrent chemotherapy. Median PRT dose was 59.4 Gy (37.5-59.4 Gy). The median time interval from the prior treatment course was 26.7 months (7-461.3). There was a rate of $13 \%$ acute $\geq$ grade 3 toxicities attributed to PRT. The median overall survival (OS) was 16.7 months (95\% CI, 4.7-36) and OS at 1 year was $67 \%$. The "in-field" failure free survival at one year was $87 \%$. The locoregional progression free survival (LPFS) and distant metastasis free survival (DMFS) at 1 year was $72 \%$ and $64 \%$ respectively. Concurrent chemotherapy was associated with a higher median survival.

Conclusions: PRT was well tolerated, resulted in prolonged clinical outcomes compared to historical controls, and should be considered as a treatment option with concurrent chemotherapy in selected patients with locally-recurrent pancreatic cancer.

Keywords: Proton; reirradiation; toxicity; pancreatic cancer; survival

Submitted Dec 18, 2016. Accepted for publication Feb 13, 2017.

doi: 10.21037/jgo.2017.03.04

View this article at: http://dx.doi.org/10.21037/jgo.2017.03.04 


\section{Introduction}

Pancreatic cancer is a morbid disease with a poor prognosis, with many patients developing both local disease progression and distant metastatic disease. Local disease control is of significant importance due to the morbidity and mortality associated with complications from obstructive symptoms, cholangitis, pain, duodenal ulceration and gastrointestinal bleeding. A rapid autopsy series has shown that up to $30 \%$ of patients die of local failure and that these patients have a genetic profile, specifically expression of SMAD4 (encoded by the DPC4 gene), which is correlated with local versus distant disease progression (1). Likewise, a significant proportion of patients develop isolated local recurrence after their initial treatment. In RTOG 9704, $28 \%$ percent of patients developed a local recurrence without evidence of distant metastases following surgery and adjuvant chemoradiation (2). Studies of SMAD4 as a prognostic biomarker correlating with improved survival (3), and local disease progression, have suggested there is a population of patients who may benefit from reestablishing local control. However, the treatment of locally-recurrent pancreatic cancer with reirradiation has been limited by the concern for toxicity associated with cumulative radiation dose and uncertainty in identifying the most appropriate patients who could benefit from aggressive local treatment.

Treating locally recurrent pancreatic cancer with a second course of radiation poses a particular risk to the surrounding abdominal organs such as the duodenum, bowel, liver, kidneys, and spinal cord which have already received a significant radiation dose in the prior treatment course. The characteristic Bragg Peak of the proton beam, with its sharp fall off, confers the ability to retreat the tumor with essentially no exit dose potentially sparing normal tissues. We have previously reported on reirradiation with proton radiotherapy (PRT) in recurrent rectal cancer and showed that it was superior in sparing bowel from radiation compared to intensity modulated radiation therapy (IMRT) with modest toxicity and complete metabolic responses in the majority of patients (4). Similarly, a locally recurrent esophageal cohort treated with proton reirradiation reported modest toxicities (maximum radiation attributed acute non-hematologic toxicity was grade 2), median overall survival (OS) of 14 months from the start of PRT, and excellent symptom palliation (5). A dosimetric comparison of proton beam radiation verses IMRT for unresectable pancreatic cancer showed decreased stomach, duodenum, and small bowel dose in the low-dose regions with proton radiation (6). In this dosimetric analysis, PRT yielded increased doses in the mid to high-dose regions to the duodenum in the intact setting, implying that patient selection may be necessary to avoid high dose to the duodenum when it is close to or included in the retreatment field. Retrospective analyses of reirradiation of locally recurrent pancreatic cancer with stereotactic body radiotherapy (SBRT) have reported median survival ranging from 5.9 to 14 months (7-9) with variable toxicity. In patients who recur locally after their initial treatment, reirradiation with PRT may offer an advantage over photon radiotherapy, allowing a second definitive dose of fractionated conformal radiation to be safely delivered with concurrent chemotherapy. In this study we report on a cohort of 15 patients with locally recurrent pancreatic cancer and report on PRT reirradiation treatment planning, acute toxicity, and survival.

\section{Methods}

\section{Patient selection}

Patients were enrolled on both an IRB-approved prospective safety and feasibility trial of PRT reirradiation (NCT01126476) $(\mathrm{n}=13)$, or an IRB approved institutional proton registry $(\mathrm{n}=2)$. All 15 patients met eligibility for the PRT reirradiation trial, including age $>18$ with histologically confirmed pancreatic adenocarcinoma, who were previously radiated at least 3 months prior and had a recurrence in the previously treated radiation field, without evidence of metastatic disease. Patients were required to have a good performance status (PS) with a KPS of $>60$ and a life expectancy of greater than or equal to 3 months. Re-biopsy was not a requirement. While it was required for PRT reirradiation eligibility that there was at least 3 months between radiation treatment courses, all but one had greater than a year between courses $(n=1$, $>6$ months; $\mathrm{n}=14,>1$ year between RT courses).

\section{Treatment planning and delivery}

Patients were 4D-CT simulated in the supine position using an immobilization device. The GTV (gross target volume) was defined as any gross disease visible on the CT treatment planning scan, as well as FDG avidity on PET-CT fused imaging used for treatment planning in 12 patients. The clinical target volume (CTV) consisted of the GTV plus any areas considered at high risk for microscopic disease 
based on the clinical judgment of the treating physician and an ITV (internal target volume) taking into account respiratory motion based on the $4 \mathrm{D}-\mathrm{CT}$ scan. Elective nodal irradiation was not included in the CTV target. A $0.5-1 \mathrm{~cm}$ margin for planning target volume PTV was used for evaluation purposes, although the ITV/CTV was used for PRT treatment planning per institutional standards. Passive scatter PRT was used in all cases. Evaluation CTscans were done every 2 weeks in the treatment position to assess for changes in anatomy, such as weight loss, variation gastric filling, or bowel gas. Feasibility of PRT treatment planning was defined as $<15 \%$ of treatments delivered using photons, completion within 10 days of the estimated date and without treatment breaks $>5$ days.

\section{Follow-up}

Toxicity was assessed using the Common Terminology Criteria for Adverse Events version 4.0. Acute events were scored as those occurring less than 90 days following the completion of PRT reirradiation. Attribution of events to proton therapy was determined by the PI in consultation with the treating physician. Locoregional progression following PRT reirradiation was defined as including both regional and local (including in-field) tumor growth documented with surveillance CT imaging and clinician reported follow-up. Local "in-field" progression was assessed as progression within the PTV.

\section{Statistical analysis}

Descriptive statistics including toxicity, time to events such as locoregional progression, local "in-field" progression and distant metastasis as well as survival data are reported. OS was defined as the number of days from the date of first PRT treatment to date of death. Locoregional progression free survival (LPFS) was defined as the number of days from date of first PRT treatment to the date of first documented incidence of locoregional progression documented on surveillance imaging. Distant metastasis free survival (DMFS) was defined as the number of days from date of first PRT treatment to first documented radiographic evidence of metastatic disease. Kaplan-Meier analysis was used to determine OS, LPFS, DMFS. Log-rank test was used to compare survival in patients receiving concurrent chemotherapy verses proton radiation alone. Data were analyzed using STATA Version 13 (StataCorp LP, College Station, TX, USA).

\section{Results}

\section{Patient demographics}

Fifteen patients with locally recurrent pancreatic cancer were treated with PRT reirradiation from 8/2010-2/2015 (Table 1). The mean age was 67 years [54-79] and the baseline performance status (PS) was ECOG $0-1$ in all patients. The median follow-up was 15.7 months [2-48] from the start of PRT and the median time interval from the prior treatment course to the start of the PRT was 26.7 months [7-90]. Thirteen patients had resections of the primary tumor as a part of their initial treatment course; 9 patients were treated with adjuvant chemoradiation and 4 patients were treated neoadjuvantly with chemoradiation. Two patients were treated with definitive chemoradiation to unresectable disease as their initial therapy. Nine patients received adjuvant Gemcitabine following their initial treatment course for a median number of 4 months [2-6]. The median time from the prior radiation treatment course to local recurrence was 17.7 months [4-86]. Twelve of the patients went over a year from the prior radiation treatment course before a local recurrence was detected. Following diagnosis of local recurrence, seven patients were treated with chemotherapy prior to receiving PRT. Four of these patients were treated with FOLFOX, with one patient switched to Gemcitabine/Abraxane and one patient switched to Capecitabine and then Gemcitabine for continued local progression (3 lines of treatment). One patient was treated with Gemcitabine and Tarceva. One patient was treated with Gemcitabine/Abraxane and one patient was treated with FOLFIRINOX and then switched to Gemcitabine for continued progression. The median number of months of systemic treatment for local recurrence was 4 months [2-18] prior to PRT. The median number of months of observation from the time of recurrence to start of PRT for patients who did not receive chemotherapy for local recurrence was 1.7 months (1.2-10).

The median CTV/ITV size treated with PRT was 71 cc [15-200]. Median PRT dose was 59.4 Gy (relative biological effectiveness, RBE) (37.5-59.4 RBE) and the median prior radiation dose was 50.4 Gy (30-59.4). One patient was treated with palliative intent to 37.5 Gy to the celiac portion of a painful recurrence, based on the large volume of disease which would need to be included if treated definitively and age. The recurrences treated with PRT were all isolated local recurrences, most commonly in the surgical bed and associated with celiac, porta-hepatic, superior mesenteric artery (SMA), and aortocaval lymph 
Table 1 Patient demographics and treatment characteristics

\begin{tabular}{|c|c|}
\hline Parameter & Data \\
\hline \multicolumn{2}{|l|}{ Age (yrs) } \\
\hline Mean & 67 [54-79] \\
\hline \multicolumn{2}{|l|}{ Performance Status } \\
\hline ECOG 0 & 5 \\
\hline ECOG 1 & 10 \\
\hline \multicolumn{2}{|l|}{ Median follow-up (months) } \\
\hline From the start of PRT & $15.7[2-48]$ \\
\hline From local recurrence & $17.2[2.4-43.7]$ \\
\hline \multicolumn{2}{|l|}{ Tumor location within pancreas (no.) } \\
\hline Head & 10 \\
\hline Body & 3 \\
\hline Tail & 2 \\
\hline \multicolumn{2}{|l|}{ Initial stage } \\
\hline Resectable & 10 \\
\hline Borderline resectable & 3 \\
\hline Unresectable & 2 \\
\hline \multicolumn{2}{|l|}{ Initial treatment course } \\
\hline Surgery with curative intent (no.) & 13 \\
\hline Ro & 10 \\
\hline $\mathrm{R} 1$ & 3 \\
\hline $\mathrm{N}+$ & 8 \\
\hline \multicolumn{2}{|l|}{ Initial radiation course (no.) } \\
\hline Neoadjuvant CRT/surgery & 4 \\
\hline Surgery/adjuvant CRT & 9 \\
\hline Definitive CRT & 2 \\
\hline \multicolumn{2}{|l|}{ Initial radiation dose (CGY) } \\
\hline Median & $5,040[4,500-5,940]$ \\
\hline \multicolumn{2}{|l|}{ Proton reirradiation course } \\
\hline Interval between radiation courses (mo.) & 26 [7-90] \\
\hline \multicolumn{2}{|l|}{ Reirradiation dose (cGy) } \\
\hline Median & $5,940[3,750-5,940]$ \\
\hline \multicolumn{2}{|l|}{ CTV volume (cc) } \\
\hline Median & $71[15-200]$ \\
\hline
\end{tabular}

Table 1 (continued)
Table 1 (continued)

\begin{tabular}{lc}
\hline Parameter & Data \\
\hline Concurrent chemotherapy (no.) & 10 \\
Capecitabine & 6 \\
5FU & 3 \\
5FU/oxaliplatin & 1 \\
\hline ECOG, Eastern Cooperative Oncology Group; PRT, proton \\
beam reirradiation; R, resection; N, Iymph nodes; CRT, \\
chemoradiation; CTV, clinical target volume; 5FU, 5-fluorouracil.
\end{tabular}

Table 2 List of local recurrences treated with proton reirradiation

\begin{tabular}{lc}
\hline Recurrent tumor location & No. of patients \\
\hline Lymph nodes & 6 \\
Celiac & 2 \\
Aortocaval & 2 \\
Porta-hepatis & 1 \\
SMA & \\
Surgical bed/primary & 2 \\
Surgical bed & 2 \\
Remnant pancreas/surgical bed & 1 \\
Duodenum/lesser curvature/surgical bed & 2 \\
Progression of primary (initial course def. CRT) & \\
\hline $\begin{array}{l}\text { SMA, superior mesenteric artery; def. CRT, definitive } \\
\text { chemoradiation. }\end{array}$
\end{tabular}

nodes (Table 2). Fourteen patients had recurrences directly within the prior radiation treatment field and one patient had a marginal nodal recurrence at the inferior boarder of the prior radiation field. Seven of the patients were symptomatic from local recurrence, reporting abdominal and back pain prior to the start of PRT.

Ten patients received concurrent 5 -fluorouracil or capecitabine-based chemotherapy with PRT. Interestingly, the five patients who did not receive concurrent chemotherapy had PS of ECOG $0-1$ and did not receive concurrent treatment for a variety of reasons independent of PS. Two patients were not offered concurrent chemotherapy due to physician preference, one patient was felt to be too heavily pretreated and had progressed on Capecitabine 
prior to the start of PRT, one patient refused, and one patient was not treated with concurrent chemotherapy since they received a hypofractionated PRT course (250 cGy per fraction).

\section{Radiation treatment}

Fourteen patients were treated with double-scatter and one with uniform scanning PRT using 2-3 co-planar proton beams arranged in order to spare the maximal amount of normal tissue and optimize robustness (Figure 1). Fields were often arranged more posteriorly (using posterior and oblique fields) to avoid anterior bowel, and laterally to spare liver or kidney, or were arranged anteriorly to spare kidney and spinal cord depending on the prior treatment course doses to normal organs at risk (OARs) and the tumor location relative to the patient's anatomy.

\section{Feasibility and toxicity}

All patients enrolled on the PRT reirradiation protocol were treated with proton plans. One patient enrolled on the proton registry, previously treated with definitive chemoradiation to unresectable disease, was treated with $35 \%$ volumetric modulated arc therapy (VMAT) to decrease the Dmax to the duodenum which was close to the target. There were no treatment breaks. Most patients tolerated PRT well with grade 1 and 2 non-hematologic acute toxicities observed (Table 3). Two patients had acute grade 3 anorexia and fatigue but recovered 1-2 months following the completion of radiation (Table 4). One patient with grade 4 toxicity likely attributed to PRT had a biliary stent placed 9 months prior to the start of PRT and was treated to the unresected primary (initial treatment was definitive chemoradiation) with a portion of the duodenum in the treatment field. This patient developed a bleeding duodenal ulcer which required intervention one week following the completion of PRT (Table 4). Another patient developed gastric outlet obstruction (GOO) from progression of disease at the end of treatment and died from a small bowel perforation following stent placement 3 days after the completion of reirradiation. Progressive disease was confirmed with biopsy at the time of stent placement and perforation may have been attributed to disease progression and instrumentation rather than radiation treatment, however this toxicity was scored as possibly relate to PRT (Table 4). There were no grade 2 or higher late nonhematologic radiation-related toxicities.

\section{Symptom palliation, tumor control and survival}

Six of seven (86\%) patients who reported pain at their initial consult reported palliation of pain during PRT and at their first follow-up. CA 19-9 was elevated in 7 patients with a median pretreatment value of 429 units/mL [62-1,000]. Following PRT, there was a $75 \%$ decrease with a median post-treatment CA 19-9 of 95 units/mL [30-66,560]. CA 19-9 remained elevated in two patients following PRT who were found to have progressive disease, one with lung metastases (post-treatment CA 19-9 was 928 units/mL) and one patient with progressive local disease and GOO (posttreatment CA $19-9$ was 66,560 units $/ \mathrm{mL}$ ) within 2 months of completing treatment.

Three patients developed isolated local progression in the treatment field and one developed lung metastases at 6.9 months and subsequent progression at 12.2 months in the treatment field (Figure 2). Two of these in-field failures developed soon after the completion of treatment; one identified at stent placement one week following the end of treatment and the other patient, who was treated to a palliative dose, with local disease progression 2 months after PRT. The other two patients had in-field progression at 12.2 and 14 months following the completion of reirradiation (Figure 2). There were three patients with locoregional failures outside of the treatment field. One patient was found to have progressive disease inferior to the field (in the SMA) toward the end of treatment and completed early at 4,500 cGy. The other two patients developed locoregional nodal progression in the porta-hepatis (5.5 and 11 months) and subsequent liver metastases. One patient remains alive and free of distant or locoregional disease and one patient is alive with liver metastases. Two patients are deceased with uptrending CA 19-9, failure to thrive, and suspected peritoneal carcinomatosis at 16.5 and 22.5 months without evidence of local progression or distant metastasis on imaging (Figure 2). Eight patients developed metastatic disease in the liver and lungs with an indolent course and median survival of 8 months $(0.5-27.8)$ from the diagnosis of metastatic disease. Only six patients were treated with chemotherapy following PRT for disease progression and the median chemotherapy-free interval in these patients was 7 months [4-17].

The median OS was 16.7 months from the start of PRT (95\% CI, $4.7-36)$ and OS at one year was $67 \% \pm 12 \%$ (Figure $3 A$ ). The "in-field" failure free survival at 1 year was $86.7 \% \pm 8.7 \%$. The LPFS and DMFS at 1 year was $72 \% \pm 12 \%$ and $64 \% \pm 13 \%$ respectively. Concurrent 

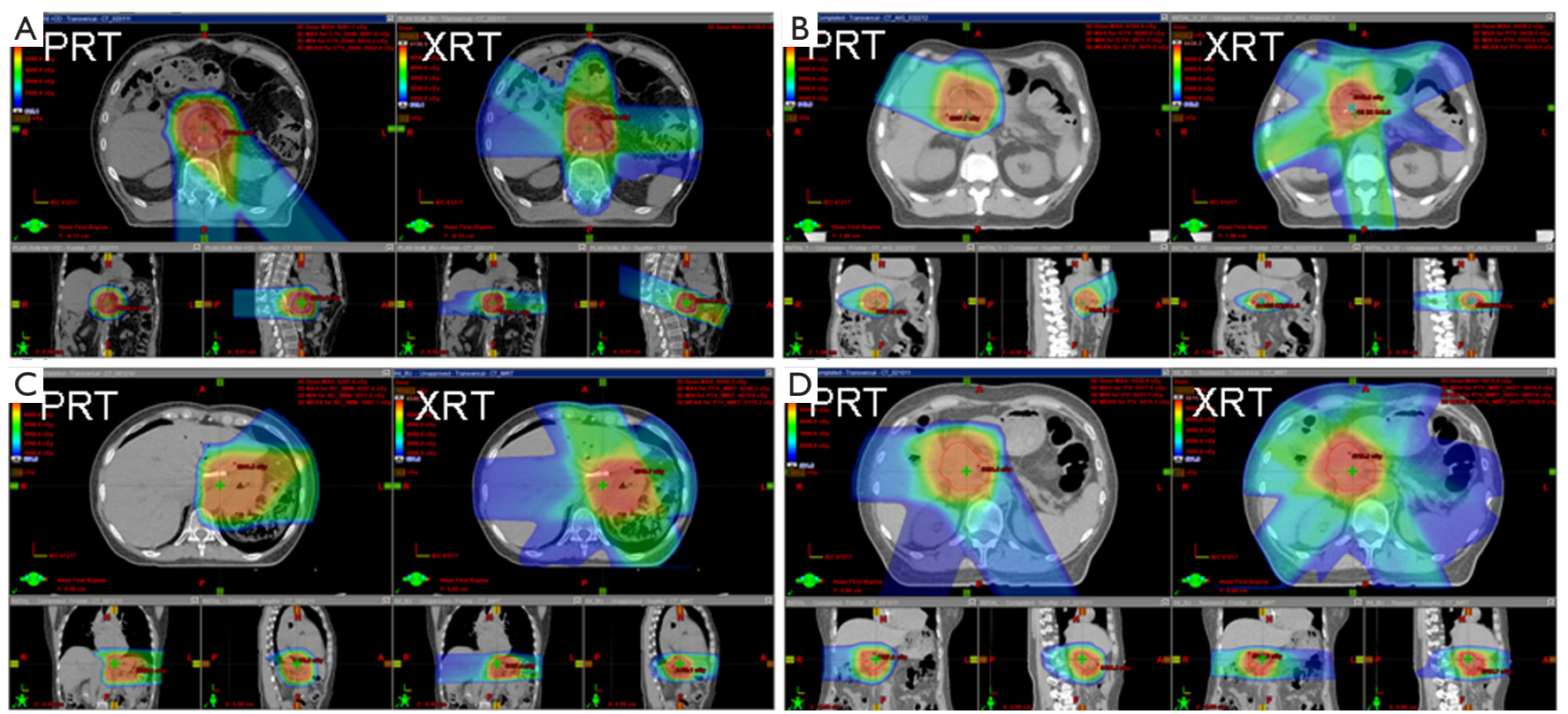

Figure 1 Representative dose distributions of PRT versus Intensity Modulated Radiation Therapy (XRT). Proton beams were chosen to maximize dose limitation to cord, kidneys, and bowel based on prior radiation fields and anatomy. (A) Two beams, posterior and posteriorlateral oblique fields to spare bowel and kidney; (B) two beams, anterior-superior and anterior-lateral oblique to spare the cord, kidneys and bowel; (C) two beams, Anterior-lateral oblique and lateral fields to spare cord and liver; (D) three beams, posterior-lateral obliques and lateral fields to spare bowel. PRT, proton reirradiation.

Table 3 List of grade 2 non-hematologic acute toxicities (CTCAE v.4.0)

\begin{tabular}{ll}
\hline Gastrointestinal & Other \\
Anorexia & Neuropathy \\
Weight loss & Hypoalbuminemia \\
Nausea & Dehydration \\
Oral mucositis & Fatigue \\
Esophagitis & \\
Diarrhea &
\end{tabular}

CTCAE v.4.0, Common Terminology Criteria for Adverse Events version 4.0 .

chemotherapy was associated with a higher MS of 22.8 months compared to a median survival of 7.6 months without chemotherapy, $\mathrm{P}=0.003$ (Figure 3B).

\section{Discussion}

In this carefully selected cohort of patients, PRT reirradiation was feasible and well-tolerated with mainly grade 1 and
Table 4 Acute toxicities with PRT alone and PRT with chemotherapy (no.)

\begin{tabular}{lcccc}
\hline \multirow{2}{*}{ Treatment } & \multicolumn{5}{c}{ Grade (CTCAE v.4.0) } \\
\cline { 2 - 5 } PRT alone $(\mathrm{n}=5)$ & 1 & 2 & 3 & $4, \geq 4$ \\
PRT + chemo. $(\mathrm{n}=10)$ & 5 & 5 & 1 & 0,1 \\
\hline
\end{tabular}

CTCAE v.4.0, Common Terminology Criteria for Adverse Events version 4.0; PRT, proton beam reirradiation; chemo, chemotherapy.

2 acute toxicities. Caution should be exercised when combining reirradiation with biliary and enteral stents and when there is a portion of the previously irradiated duodenum within the reirradiation treatment field. The need for an enteral or biliary stent in these patients is likely a surrogate of duodenal involvement or disease in the pancreatic head in close proximity to the duodenum. This could place the duodenum within the reirradiation field, putting these patients at higher risk for bowel ulceration and perforation. Likewise, these patients with duodenum in the retreatment field, would not be SBRT candidates, 

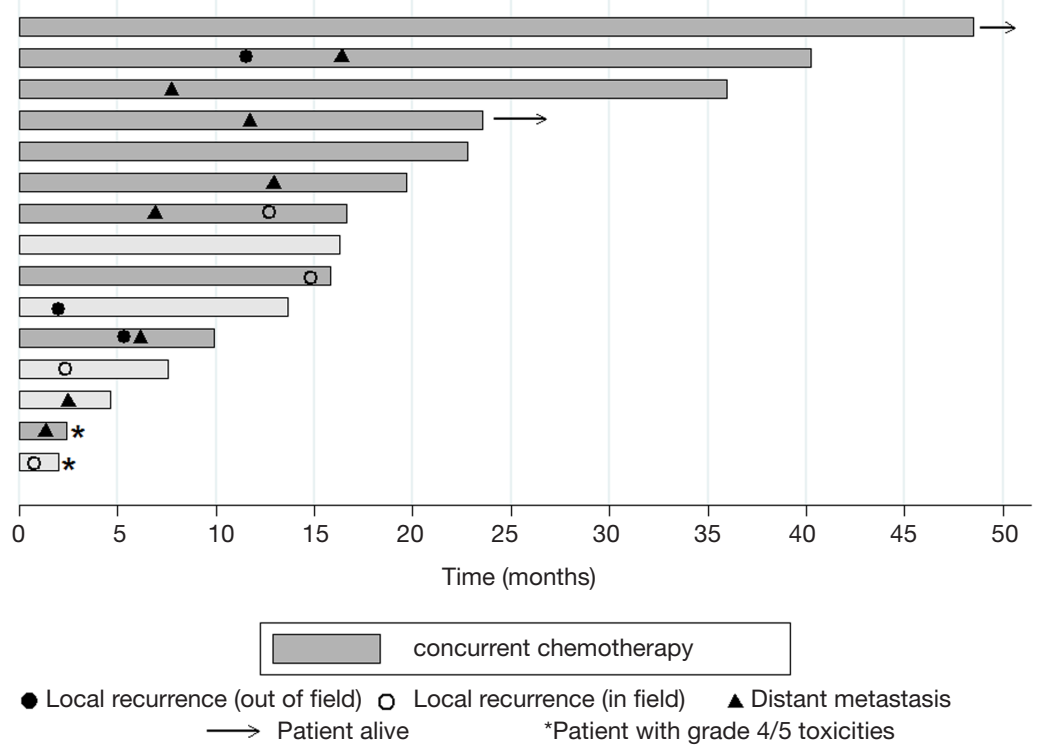

Figure 2 Time to event outcomes in 15 patients treated with proton reirradiation. A swimmers plot from start of PRT to time of local progression, distant metastasis and survival. Each bar represents a single patient.

A

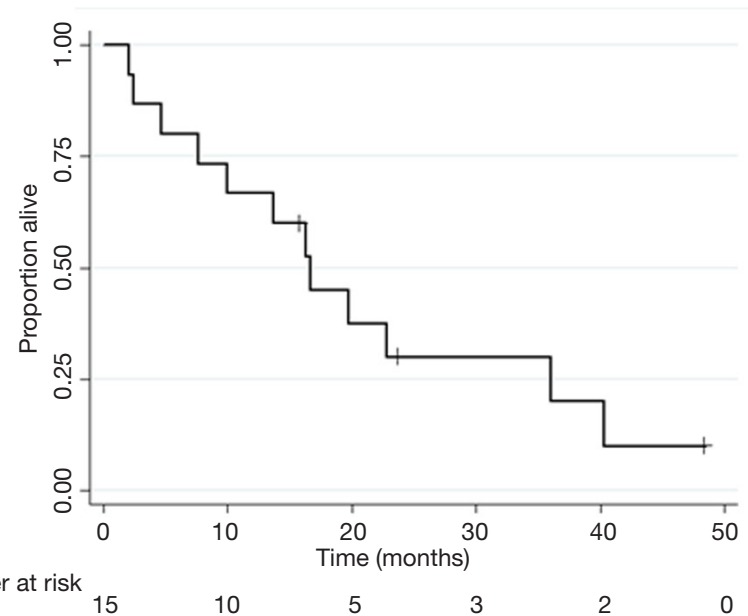

B

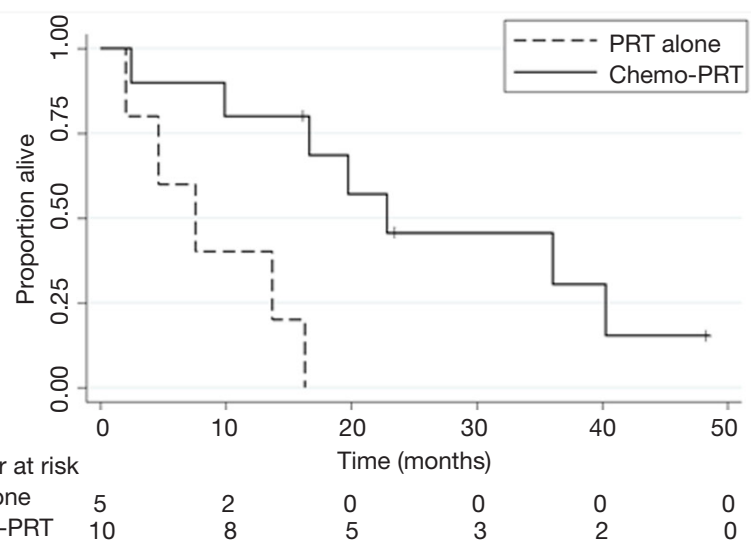

Figure 3 Kaplan Meier analysis. (A) OS (median survival =16.7 moths, 95\% CI, 4.7-36, cross-hair = censored); (B) OS in patients with and without concurrent chemotherapy by log-rank test, $\mathrm{P}=0.003$. PRT, proton radiotherapy.

and potentially at even greater risk for $\geq$ grade 4 toxicities with a higher dose per fraction used in the reported SBRT reirradiation studies.

The median survival of 16.7 months with PRT reirradiation is comparable or longer than the previously published survival of reirradiation for locally recurrent pancreatic cancer and is a feasible option for retreatment in this setting (9). A concern for reirradiation is the potential for toxicity to normal tissues. A recent retrospective review of 30 patients with locally recurrent pancreatic cancer treated with SBRT reirradiation reported $10 \%$ acute grade 3 toxicities including pain, bleeding, and vomiting, as well as a $7 \%$ rate of late bowel-obstructions (9). Excluding the patient who died from a perforation likely secondary to 
disease progression and stent placement, our cohort had a rate of $13 \%$ acute $\geq$ grade 3 toxicities of fatigue, anorexia, and bleeding which resolved following the completion of treatment. There were no $\geq$ grade 3 late toxicities, suggesting PRT may reduce the probability of late complications compared to SBRT. Additionally, the ability to give concurrent chemotherapy with PRT reirradiation is significant as pancreatic cancer is a systemic disease and these patients remain at high risk of developing distant metastases. We acknowledge that patients who are able to tolerate and receive concurrent chemotherapy may be a selected more favorable patient population. However, all of our patients similarly had a significant interval from the prior radiation course to the local recurrence and an excellent PS. In these patients, concurrent chemotherapy was associated with a median survival of 23 months, increased from median survival ranging from 6-14 months in prior reports of reirradiation.

Patients with locally recurrent pancreatic cancer have limited therapeutic treatment options after prior external beam radiation. The standard of care for pancreatic cancer with local recurrence or progression following definitive treatment is chemotherapy alone (10-12). There is little consensus on the optimal chemotherapy regimen and selection is usually based on the prior treatment history and PS with a median survival between 6-9 months. Surgery is often not a feasible option due to the amount of fibrosis from the prior radiation treatment course, poor PS, and inability to undergo an R0 resection. Surgical series of locally recurrent pancreas cancer have reported median survivals of 7.4-11.4 months $(13,14)$. However, postoperative morbidity and mortality was significant at 20-40\% (14). The median survival we report in this small group of patients with PRT reirradiation is relatively long compared to locally recurrent pancreatic cancer patients treated with chemotherapy alone or surgical resection.

The median treatment dose in this study (59.4 Gy) is often used in the definitive setting for gross disease and accordingly the median survival of 16.4 months in our heavily pretreated cohort is in the range reported for locally advanced pancreatic cancer treated with definitive chemoradiation in the primary treatment setting. Historical retrospective studies from the Mayo Clinic and Johns Hopkins report median survival for definitive chemoradiation ranging from 11-20 months (15). A phase I/II study from the University of Michigan evaluated high dose conformal IMRT with concurrent gemcitabine reported a median survival of 14.8 months (16). Most recently, the LAP-07 trial reported median survivals of 16.5 months with chemotherapy alone and 15.2 months with chemoradiation in patients with locally advanced pancreatic cancer, with no significant difference between the two regimens (17). Despite these results, many patients develop local only progression resulting in significant morbidity and mortality. The combination of Gemcitabine/ nab-paclitaxel has shown improved survival in the metastatic setting and is being evaluated in locally advanced patients (18). As our sequential systemic therapies improve but local recurrence remains a problem, we will ultimately select for a group of patients that are heavily pretreated and in need of local palliation and treatment. The patients in our cohort, developing isolated local progression and a late indolent metastatic course, benefited from a second course of aggressive combined modality treatment.

This study selected patients with local recurrence who did not develop metastatic disease for retreatment, but in future studies biomarkers are needed to help select these patients earlier in the course of their disease for aggressive local treatment. We may utilize known molecular markers such as SMAD4, shown to correlate with locally recurrent disease. Additionally, PRT reirradiation may not offer a significant advantage over IMRT or VMAT when there is gross disease in close proximity to the duodenum. This is the case when the initial treatment was definitive chemoradiation to unresectable disease and there is duodenum close to or within the PTV for a second treatment course. We have previously described the dosimetric advantages of protons in lowering the volume of bowel and stomach treated in the low-dose region, however, due to decreased conformality of passively scattered protons, there can be an increased volume of duodenum in the high-dose region depending on the proximity to the target (6). In these cases, combined photon/proton plans may offer an advantage in lowering the high dose to the duodenum while still maintaining low dose to the other OARs. Patients also reported improvements in pain control and a prolonged chemotherapy free interval following PRT. Future studies and clinical trials of proton reirradiation for locally recurrent pancreatic cancer may integrate biomarkers, technical advances in PRT (such as pencil beam scanning), and quality of life endpoints to better define patients who may benefit from reirradiation with a favorable therapeutic window.

\section{Conclusions}

This series reports on a cohort of pancreatic cancer patients 
with local recurrence following prior definitive treatment with surgery and chemoradiation reirradiated with PRT. A relatively long median survival time was observed with modest acute and late toxicities and PRT is a reasonable treatment option for patients that are candidates for aggressive local treatment. Proton therapy with concurrent chemotherapy should be considered a viable treatment option in patients with locally recurrent pancreatic cancer, without evidence of metastatic disease, with an interval of at least 1 year after previous radiation.

\section{Acknowledgements}

The authors acknowledge Caitlin Schonewolf MD and David Guttmann MD for statistical assistance. This work was supported by institutional research funding at University of Pennsylvania (NCT01126476).

\section{Footnote}

Conflicts of Interest: The authors have no conflicts of interest to declare.

Ethical Statement: The data presented in this manuscript was acquired in accordance with the policies of the Institutional Review Board at the University of Pennsylvania (NO. 811027).

\section{References}

1. Iacobuzio-Donahue CA, Fu B, Yachida S, et al. DPC4 gene status of the primary carcinoma correlates with patterns of failure in patients with pancreatic cancer. J Clin Oncol 2009;27:1806-13.

2. Regine WF, Winter KA, Abrams R, et al. Fluorouracilbased chemoradiation with either gemcitabine or fluorouracil chemotherapy after resection of pancreatic adenocarcinoma: 5-year analysis of the U.S. Intergroup/ RTOG 9704 phase III trial. Ann Surg Oncol 2011;18:1319-26.

3. Tascilar M, Skinner HG, Rosty C, et al. The SMAD4 protein and prognosis of pancreatic ductal adenocarcinoma. Clin Cancer Res 2001;7:4115-21.

4. Berman AT, Both S, Sharkoski T, et al. Proton Reirradiation of Recurrent Rectal Cancer: Dosimetric Comparison, Toxicities, and Preliminary Outcomes. International Journal of Particle Therapy 2014;1: 2-13.
5. Fernandes A, Berman AT, Mick R, et al. A Prospective Study of Proton Beam Reirradiation for Esophageal Cancer. Int J Radiat Oncol Biol Phys 2016;95:483-7.

6. Thompson RF, Mayekar SU, Zhai H, et al. A dosimetric comparison of proton and photon therapy in unresectable cancers of the head of pancreas. Med Phys 2014;41:081711.

7. Lominska CE, Unger K, Nasr NM, et al. Stereotactic body radiation therapy for reirradiation of localized adenocarcinoma of the pancreas. Radiat Oncol 2012;7:74.

8. Wild AT, Hiniker SM, Chang DT, et al. Re-irradiation with stereotactic body radiation therapy as a novel treatment option for isolated local recurrence of pancreatic cancer after multimodality therapy: experience from two institutions. J Gastrointest Oncol 2013;4:343-51.

9. Dagoglu N, Callery M, Moser J, et al. Stereotactic Body Radiotherapy (SBRT) Reirradiation for Recurrent Pancreas Cancer. J Cancer 2016;7:283-8.

10. Lee MG, Lee SH, Lee SJ, et al. 5-Fluorouracil/leucovorin combined with irinotecan and oxaliplatin (FOLFIRINOX) as second-line chemotherapy in patients with advanced pancreatic cancer who have progressed on gemcitabinebased therapy. Chemotherapy 2013;59:273-9.

11. Rahma OE, Duffy A, Liewehr DJ, et al. Second-line treatment in advanced pancreatic cancer: a comprehensive analysis of published clinical trials. Ann Oncol 2013;24:1972-9.

12. Gresham GK, Wells GA, Gill S, et al. Chemotherapy regimens for advanced pancreatic cancer: a systematic review and network meta-analysis. BMC Cancer 2014;14:471.

13. Kleeff J, Reiser C, Hinz U, et al. Surgery for recurrent pancreatic ductal adenocarcinoma. Ann Surg 2007;245:566-72.

14. Zacharias T, Oussoultzoglou E, Jaeck D, et al. Surgery for recurrence of periampullary malignancies. J Gastrointest Surg 2009;13:760-7.

15. Hsu CC, Herman JM, Corsini MM, et al. Adjuvant chemoradiation for pancreatic adenocarcinoma: the Johns Hopkins Hospital-Mayo Clinic collaborative study. Ann Surg Oncol 2010;17:981-90.

16. Ben-Josef E, Schipper M, Francis IR, et al. A phase I/II trial of intensity modulated radiation (IMRT) dose escalation with concurrent fixed-dose rate gemcitabine (FDR-G) in patients with unresectable pancreatic cancer. Int J Radiat Oncol Biol Phys 2012;84:1166-71

17. Hammel P, Huguet F, van Laethem JL, et al. Effect 
of Chemoradiotherapy vs Chemotherapy on Survival in Patients With Locally Advanced Pancreatic Cancer Controlled After 4 Months of Gemcitabine With or Without Erlotinib: The LAP07 Randomized Clinical
Trial. JAMA 2016;315:1844-53.

18. Von Hoff DD, Ervin T, Arena FP, et al. Increased survival in pancreatic cancer with nab-paclitaxel plus gemcitabine. N Engl J Med 2013;369:1691-703.

Cite this article as: Boimel PJ, Berman AT, Li J, Apisarnthanarax S, Both S, Lelionis K, Larson GL, Teitelbaum U, Lukens JN, Ben-Josef E, Metz JM, Plastaras JP. Proton beam reirradiation for locally recurrent pancreatic adenocarcinoma. J Gastrointest Oncol 2017;8(4):665-674. doi: 10.21037/ jgo.2017.03.04 